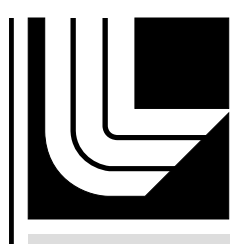

LAWRENCE LIVERMORE N A TIO NAL LABORATORY

\title{
Theory of (3He,alpha) surrogate reactions for deformed uranium nuclei
}

I. Thompson, J. E. Escher

November 9, 2006 
This document was prepared as an account of work sponsored by an agency of the United States Government. Neither the United States Government nor the University of California nor any of their employees, makes any warranty, express or implied, or assumes any legal liability or responsibility for the accuracy, completeness, or usefulness of any information, apparatus, product, or process disclosed, or represents that its use would not infringe privately owned rights. Reference herein to any specific commercial product, process, or service by trade name, trademark, manufacturer, or otherwise, does not necessarily constitute or imply its endorsement, recommendation, or favoring by the United States Government or the University of California. The views and opinions of authors expressed herein do not necessarily state or reflect those of the United States Government or the University of California, and shall not be used for advertising or product endorsement purposes.

This work was performed under the auspices of the U.S. Department of Energy by University of California, Lawrence Livermore National Laboratory under Contract W-7405-Eng-48. 


\title{
Theory of $\left({ }^{3} \mathrm{He}, \alpha\right)$ surrogate reactions for deformed uranium nuclei
}

\author{
Ian Thompson and Jutta E. Escher \\ Lawrence Livermore National Laboratory, Livermore, CA 94550
}

November 8, 2006

\begin{abstract}
We present the one-step theory of neutron-pickup transfer reactions with ${ }^{3} \mathrm{He}$ projectiles on ${ }^{235} \mathrm{U}$ and ${ }^{238} \mathrm{U}$. We find all the neutron eigenstates in a deformed potential, and use those in a given energy range for $\left({ }^{3} \mathrm{He}, \alpha\right)$ DWBA pickup calculations to find the spin and parity distributions of the residual target nuclei. A simple smoothing convolution is used to take into account the spreading width of the single-neutron hole states into the more complicated compound nuclear states. We assume that the initial target is an even-even rotor, but can take into account spectator neutrons outside such a rotor by recombining their spin and parity at the end of the calculations.
\end{abstract}

\section{Introduction}

Results from recent Surrogate experiments, aimed at determining (n,f) cross sections for actinide targets, have shown reasonable agreement with direct measurements, where available. Discrepancies between the extracted and expected cross sections are, to a large extent, due to differences in the spins (and parities) of the compound nuclear states populated in the n-induced and Surrogate (direct) reactions, respectively [1]. This " $J{ }^{\pi}$ mismatch" has been the motivation for some recent experiments at LBNL [2] that focus on transfer reactions with ${ }^{3} \mathrm{He}$ projectiles on ${ }^{235} \mathrm{U}$ and ${ }^{238} \mathrm{U}$. Here, we perform some calculations that will aid the interpretation of these experiments and allow us to assess the feasibility of adapting the Surrogate technique to situations where the $J^{\pi}$ mismatch becomes non-negligible. Specifically, we provide calculations of $\left({ }^{3} \mathrm{He}, \alpha\right)$ transfer reactions on ${ }^{235} \mathrm{U}$ and ${ }^{238} \mathrm{U}$. We summarize and classify the single-particle and collective levels of each core nucleus, and show how to simplify the calculations using spectator approximations. We present absolute $\sigma\left(J^{\pi}\right)$ distributions for the ${ }^{234} \mathrm{U}^{*}$ and ${ }^{237} \mathrm{U}^{*}$ systems, as will be needed in the future to compare this calculation of $\left({ }^{3} \mathrm{He}, \alpha\right)$ surrogate reaction cross sections to data from the experiments carried out at LBNL.

\section{Theory}

We first have to calculate all the neutron states in ${ }^{235} \mathrm{U}$ and ${ }^{238} \mathrm{U}$ using a deformed mean field for these nuclei. We will assume that the neutron pickup in the $\left({ }^{3} \mathrm{He}, \alpha\right)$ surrogate reaction simply removes one of the neutrons from an occupied level in the target, leaving a hole in one of the neutron states. If the targets were spherical, then the neutron eigenstates $l_{i} s j_{i}$ in the spherical mean field would all be filled up to the Fermi level, and occupied with $2 j_{i}+1$ nucleons. We are now however, considering a more general 
model in which the mean field is deformed, so that states of a given total angular momentum may contain superpositions of different orbital $l$ values.

Axial deformations are described by allowing the nuclear surface and its potentials to vary according to the body-fixed intrinsic coordinates $\left(\theta^{\prime}, \phi^{\prime}\right)$ as

$$
R\left(\theta^{\prime}, \phi^{\prime}\right)=R_{0}\left[1+\sum_{k} \beta_{k} Y_{k 0}\left(\theta^{\prime}, \phi^{\prime}\right)\right] .
$$

where the $\beta_{k}$ are the fractional deformations. The deformation lengths are $\delta_{k}=\beta_{k} R_{0}$.

There are two methods for calculating these deformed neutron eigenstates. The first is to use a bodyfixed coordinate system to describe an axially deformed potential, and then find eigenstates in this potential for fixed projection $K$ along the axis of symmetry. This method is described by Bang et al [3], and used in several programs $[4,5,6]$. To be used in a transfer calculation, these $K$-labelled eigenstates have still to be rotated into the space-fixed coordinate system, by the method of [7]. Coriolis couplings may also mix different $K$ eigenstates, and the energy of rotational motion has to be added by hand.

The second method is to use a particle-rotor model, in which the motion of neutron is explicitly coupled to a set of core states $\left(0^{+}, 2^{+}, 4^{+} \ldots I_{\max }\right)$ in their rotational band. The couplings between states are calculated using a rotational model, in which the potential depends on the distance $r-R\left(\theta^{\prime}, \phi^{\prime}\right)$ of the neutron to the deformed surface. The couplings, not yet the wave functions, are rotated into space fixed coordinates. In this method the rotational energies easily enter via the core energies, and Coriolis effects are automatically included since we use directly the space-fixed coordinate system. The disadvantage is that we have to expand on many core states up to $I_{\max }$, but with suitable numerical methods this may be easily accomplished. We adopt this second method here.

We expand the wave functions for state $\left|J^{\pi} M ; E_{i}\right\rangle$ at energy $E_{i}$ in terms of core states $\phi_{I}(\xi)$, with $\xi=\left(\theta^{\prime}, \phi^{\prime}\right)$ representing the internal core structure, as

$$
\left.\left|J^{\pi} M ; E_{i}\right\rangle=\sum_{l j I} \chi_{l j I}^{J E_{i}}(r)\left\{\left[Y_{l}(\hat{r}) \otimes X_{1 / 2}\right]^{j} \otimes \phi_{I}(\xi)\right)\right\}^{J M}
$$

The unknown radial wave functions $\chi_{l j I}^{J E_{i}}(r) \equiv \chi_{\gamma}^{J E_{i}}(r)$ satisfy the coupled equations

$$
\begin{array}{r}
\left(-\frac{\hbar^{2}}{2 \mu}\left[\frac{d^{2}}{d r^{2}}-\frac{l(l+1)}{r^{2}}\right]+V_{\gamma \gamma}^{J}(r)-E+\epsilon_{I}\right) \chi_{\gamma}^{J E_{i}}(r)=-\sum_{\gamma^{\prime} \neq \gamma} V_{\gamma \gamma^{\prime}}^{J}(r) \chi_{\gamma^{\prime}}^{J}(r), \\
\text { where } V_{\gamma \gamma^{\prime}}^{J}(r)=\left\langle\left[Y_{l}(\hat{r}) X_{1 / 2}\right]^{j} \phi_{I}(\xi)\left|V_{n c}\left(r, \theta^{\prime}, \phi^{\prime}\right)\right|\left[Y_{l^{\prime}}(\hat{r}) X_{1 / 2}\right]^{j^{\prime}} \phi_{I^{\prime}}(\xi)\right\rangle
\end{array}
$$

and $V_{n c}$ is the potential between the neutron and the deformed core that depends on the distance $r-$ $R\left(\theta^{\prime}, \phi^{\prime}\right)$.

To solve this set of coupled equations we use the Lagrange mesh method of Baye et al $[9,10,11]$.

\subsection{Calculation of ${ }^{235,238} \mathrm{U}$ eigenstates}

For the potential $V_{n c}$ between the neutron and the uranium core we use the Woods-Saxon parameters from [12], namely radii of $r_{0}=1.25 \mathrm{fm}$ and diffusenesses of $a=0.65 \mathrm{fm}$ for central and spin-orbit parts. We choose a spin-orbit strength of $9.543 \mathrm{MeV}$, and a central depth adjusted to reproduce the correct Fermi level in a simple filling approximation. The sensitivity to these parameters could be used to portray the 
uncertainties in the final predictions. For the quadrupole deformation, we start by using $\beta_{2}=0.240$ for ${ }^{235} \mathrm{U}$ and $\beta_{2}=0.242$ for ${ }^{238} \mathrm{U}$, from [13]. The Fermi levels $E_{F}$ are $-5.30 \mathrm{MeV}$ for ${ }^{235} \mathrm{U}$ and $-6.15 \mathrm{MeV}$ for ${ }^{238} \mathrm{U}$.

After diagonalisation, there are many more deformed states than in a spherical potential, as here the single-particle strength is fragmented by the core couplings. Furthermore, each intrinsic state (if described in the body-fixed coordinate system) will now have all its rotational excitations included in our model.

Each particular $l j I$ part of a neutron state is fractionally occupied, and these fractions have to be multiplied by $2 J+1$ and added cumulatively to see how $N$ neutrons occupy all the levels up to the Fermi level. Since each deformed neutron eigenstate $\left|J^{\pi} M ; E_{i}\right\rangle$ is a superposition of core states $I=0 \cdots I_{\max }$, to find the cumulative occupation in (for example) the $I=0$ ground state of the system, we have to accumulate the norms $P(E)$ of the overlaps of each deformed superposition with core state $I$, namely the norms of

$$
\left\langle\phi_{0} \mid J^{\pi} M ; E_{i}\right\rangle=\chi_{l J}^{J E_{i}}(r)\left[Y_{l}(\hat{r}) \otimes X_{1 / 2}\right]^{J},
$$

as

$$
P(E)=\sum_{E_{i}<E} N_{0}\left(E_{i}\right) \text { where } N_{0}\left(E_{i}\right)=\left|\left\langle\phi_{0} \mid J^{\pi} M ; E_{i}\right\rangle\right|^{2} .
$$

We adjust the central potential so that $P\left(E_{F}\right) \simeq 146$ for ${ }^{238} \mathrm{U}$ and 143 for ${ }^{235} \mathrm{U}$.

\subsection{Spectator spins for odd targets}

For an even target such as ${ }^{238} \mathrm{U}$ that is not rotationally excited, when a hole is created by a pickup reaction the spin of the resulting nucleus is simply the spin of the hole state, namely the angular momentum of the neutron before it was removed. A rotationally unexcited ${ }^{238} \mathrm{U}$ is in its $I=0$ state, so the spin of the resulting ${ }^{237} \mathrm{U}^{*}$ nucleus will be just $j$ if a neutron is removed from component $l j 0$ of state $\left|J^{\pi} M ; E_{i}\right\rangle$, with $J=j$.

For an odd target such as ${ }^{235} \mathrm{U}$ which has a ground state spin of $s=7 / 2^{-}$, strictly speaking this spin should be coupled with the spins of all removed-neutron hole states in order to give the total spin of ${ }^{234} \mathrm{U}^{*}$. However, if the g.s. spin is entirely from an $f_{7 / 2}$ neutron, then this neutron does not have any significant effects on the dynamics of the pickup reaction. Because that neutron makes only very small contributions to the energy, deformation and moment of inertia of ${ }^{235} \mathrm{U}$, it should be a good approximation to neglect all such contributions. Thus it should be sufficiently accurate to treat that $s=7 / 2^{-}$as a spectator spin, and ignore it for calculating the deeper hole states and for calculating the initial pickup cross section. What we have to do is to recouple $s$ to final nuclear spins after the transfer reaction to spin state $J$, and distribute the cross sections according to the number of $m$-substates. That is, we introduce the factor

$$
f\left(J^{\prime}, J, s\right)=\frac{2 J^{\prime}+1}{\sum_{I=|J-s|}^{J+s}(2 I+1)}=\frac{2 J^{\prime}+1}{(J+s)(J+s+2)-(|J-s|-1)(|J-s|+1)},
$$

that gives the weight with which a calculated cross section for the removal of a $J=j$ neutron contributes to the production of a final spin- $J^{\prime}$ state, where $\vec{J}^{\prime}=\vec{J}+\vec{s}$ includes the spectator spin.

\section{$2.3 \mathrm{U}\left({ }^{3} \mathrm{He},{ }^{4} \mathrm{He}\right)$ neutron pickup calculations}

For the $\left({ }^{3} \mathrm{He},{ }^{4} \mathrm{He}\right.$ ) overlap we use a zero-range vertex with coupling constant $D_{0} \sim-300 \mathrm{MeV} \mathrm{fm}{ }^{3 / 2}$ (finite range effects can easily be included, but take much longer to calculate). Our initial approach in this project 

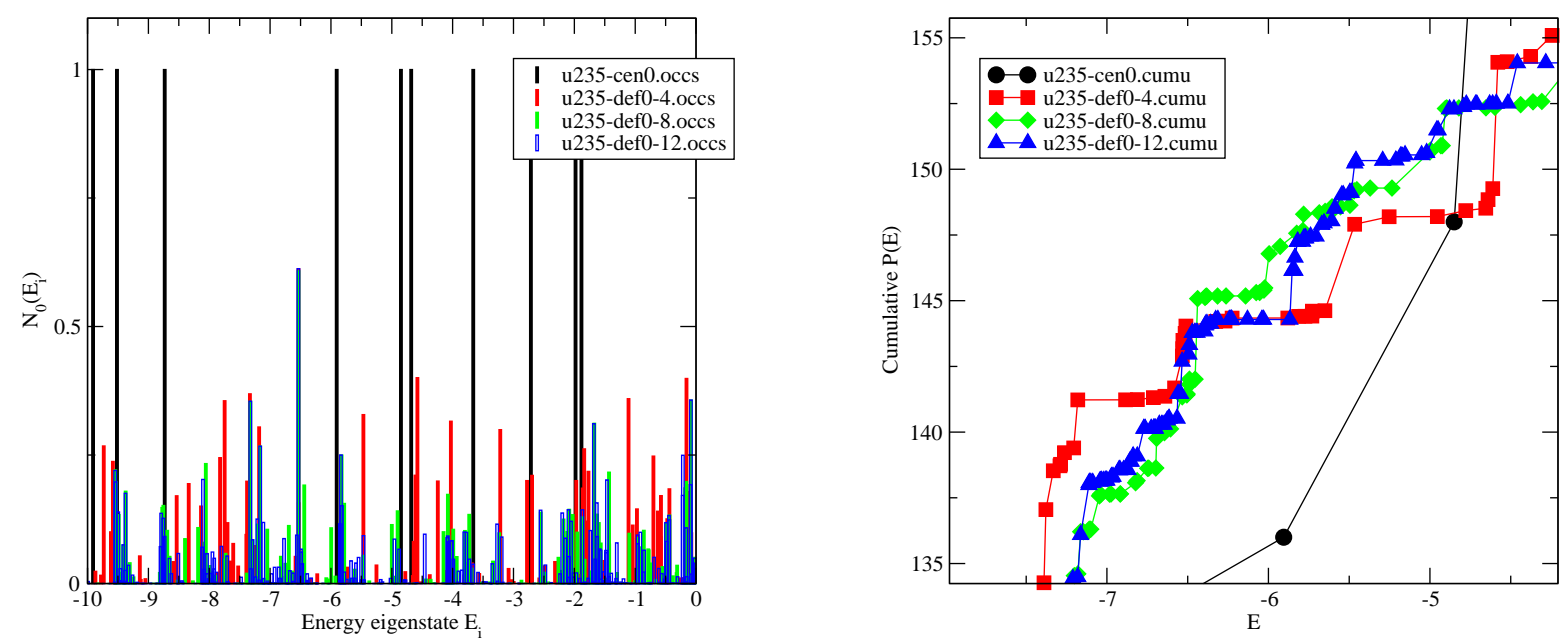

Figure 1: Left: $N_{0}\left(E_{i}\right)$ for the neutron eigenstates in ${ }^{235} \mathrm{U}$. A central potential gives the black single-particle states, and deformed models give red, blue, and green with 0-4, 0-8, and 0-12 core states respectively. Right: corresponding cumulative occupation numbers near the Fermi surface.

is to begin with the one-step Distorted Wave Born Approximation (DWBA) for the reaction. That is, we neglect any rotational excitation of the target by the incoming ${ }^{3} \mathrm{He}$, and any rotational or single-particle excitation or de-excitation by the outgoing ${ }^{4} \mathrm{He}$. So, for a one-step calculation with initial target state of $0^{+}$, we only need overlaps of deformed states with the $0^{+}$core state, namely the $\left\langle\phi_{0} \mid J^{\pi} M ; E_{i}\right\rangle$ overlap wave functions of Eq. (4).

\subsection{Spreading widths}

The DWBA pickup calculations determine the cross sections $\sigma\left(J^{\pi}, E_{i}\right)$ at the discrete energies $E_{i}$ of the hole states. In fact, these hole energies are not the true states of the compound nucleus, but are only the 'doorway states' through which the statistical process begin, which lead to the compound states. This is a 'spreading' process, and the strength of this process is described by a 'spreading width' $\Gamma_{s}$ such that the lifetime of the doorway state is $\hbar / \Gamma_{s}$. The effect of this spreading on our cross sections is described by convoluting our discrete $E_{i}$ peaks with a Lorentzian $L\left(E-E_{i}, \Gamma_{s}\left(E-E_{F}\right)\right)$ of width $\Gamma_{s}\left(E-E_{F}\right)$. The spreading width is a function of the distance from the Fermi level $E_{F}$. Brown and Rho [14] have parameterized the spreading width as $\Gamma_{s}(e)=24 e^{2} /\left(e^{2}+500\right)$ for $\Gamma$ and $e$ in $\mathrm{MeV}$. We use the Lorentzian spreading function

$$
L\left(E-E_{i}, \Gamma_{s}\right)=\frac{1}{\pi} \frac{\Gamma_{s} / 2}{\left(E-E_{i}\right)^{2}+\Gamma_{s}^{2} / 4}
$$

\section{Results}

The neutron eigenstates were found with PLUTO [8] in the deformed Woods-Saxon potential well with parameters given above, and the overlaps of these eigenstates with the non-excited core state are shown 


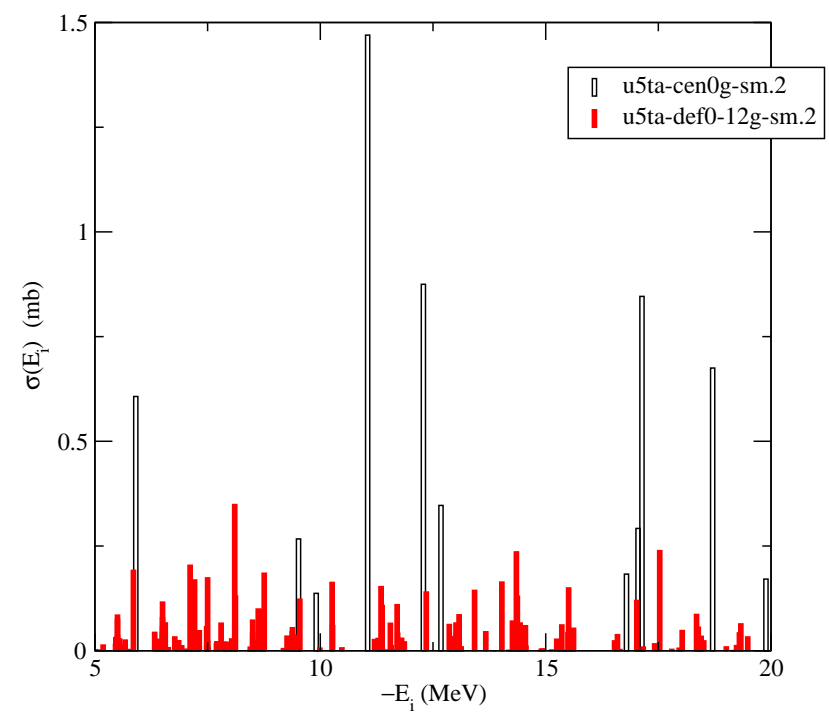

Figure 2: Cross sections $\sigma\left(J^{\pi}, E_{i}\right)$ for all hole states at $-E_{i}$, for all possible spin/parities $J^{\pi}$ in the ${ }^{235} \mathrm{U}\left({ }^{3} \mathrm{He}, \alpha\right)$ reaction. The black histogram shows the results for spherical nuclei, and the red bars for the deformed case.
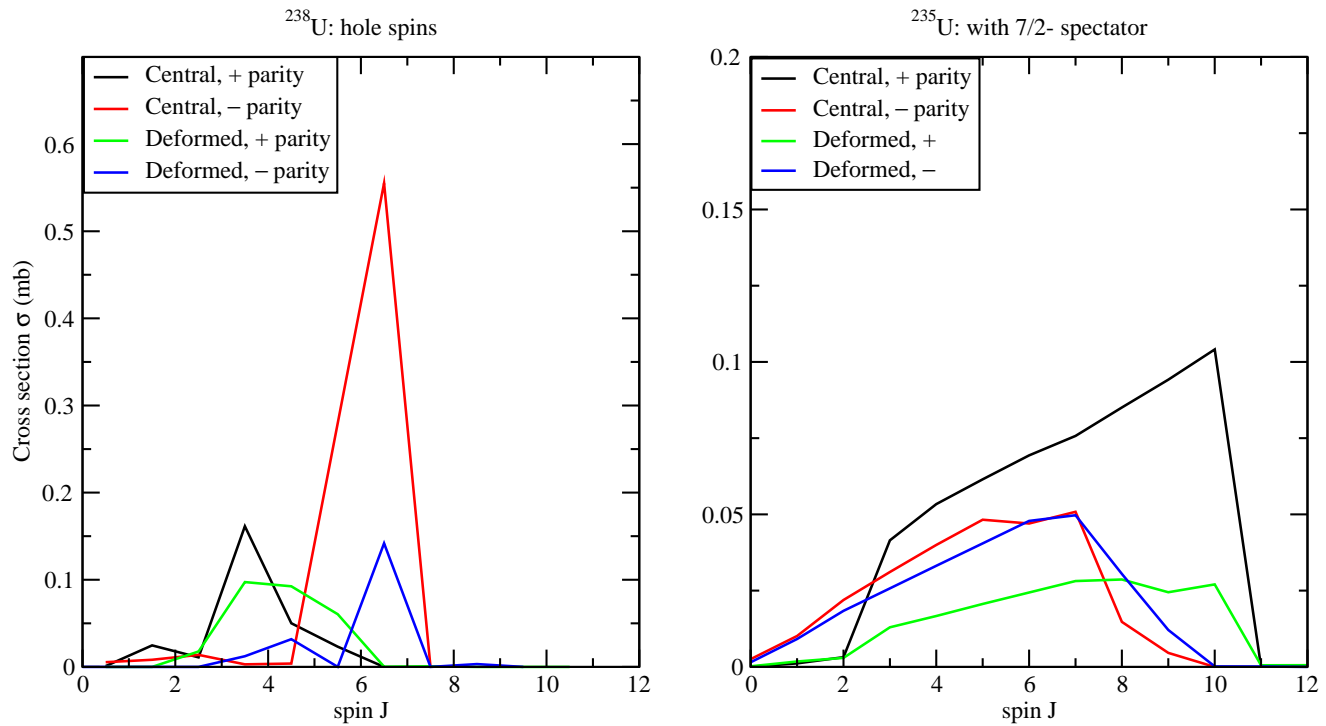

Figure 3: Spin distributions after pickup reactions for $E=11 \mathrm{MeV}$, just above the neutron emission threshold. Left: Spin distributions of ${ }^{237} \mathrm{U}^{*}$ produced in the ${ }^{238} \mathrm{U}\left({ }^{3} \mathrm{He}, \alpha\right)$ reaction, with the strong $13 / 2^{-}$ cross section in the central case. Right: Similarly for ${ }^{234} \mathrm{U}^{*}$ after the ${ }^{235} \mathrm{U}\left({ }^{3} \mathrm{He}, \alpha\right)$ reaction, for which the $7 / 2^{-}$gs spin smears out the distributions according to Eq. (6) and changes the parity. 


\begin{tabular}{c|ccc|ccc}
\hline Pair & $\begin{array}{c}V \\
\mathrm{MeV}\end{array}$ & $\begin{array}{c}r_{0} \\
\mathrm{fm}\end{array}$ & $\begin{array}{c}a \\
\mathrm{fm}\end{array}$ & $\begin{array}{c}W \\
\mathrm{MeV}\end{array}$ & $\begin{array}{c}r_{i} \\
\mathrm{fm}\end{array}$ & $\begin{array}{c}a_{i} \\
\mathrm{fm}\end{array}$ \\
\hline${ }^{3} \mathrm{He}$ & 154.00 & 1.20 & 0.72 & 36.00 & 1.40 & 0.88 \\
${ }^{4} \mathrm{He}$ & 159.26 & 1.245 & 0.771 & 14.437 & 1.57 & 0.584 \\
\hline
\end{tabular}

Table 1: Optical potentials used for the $\left({ }^{3} \mathrm{He}, \alpha\right)$ DWBA transfer calculation.

on the left side of Fig. 1. On the right side of this figure are portrayed the cumulative neutron numbers in the region of the Fermi surface of the two Uranium isotopes. We conclude that for the overlap with just the ground state of the rotor, Eq. (4), the calculations are sufficiently accurate once rotor states up to $8^{+}$ are included.

Using these one-channel overlaps with the gs of the rotor, $\left({ }^{3} \mathrm{He}, \alpha\right)$ DWBA pickup calculations were performed with FRESCO [15] for incident ${ }^{3} \mathrm{He}$ at $42 \mathrm{MeV}$ (lab), using the entrance and exit optical potentials given in Table 1. The resulting cross sections to the discrete hole states - the $\sigma\left(J^{\pi}, E_{i}\right)$ - are shown in Fig. 2.

Folding with the spreading width using the kernel function of Brown and Rho given by Eq. (7) gives the spin and parity distributions shown on the left side of Fig. 3, for ${ }^{238} \mathrm{U}$, an even target. As expected, the deformed case gives a smoother distribution of cross sections, without the very strong $13 / 2^{-}$cross section peak seen in the spherical case.

For the odd target of ${ }^{235} \mathrm{U}$, there is a non-zero ground state spin $\left(7 / 2^{-}\right)$. In our spectator approximation for this spin, its effect is to broaden the $J$ distribution around $s=7 / 2$ according to the kernel function of Eq. (6). The resulting distributions are shown on the right hand side of Fig. 3, and would be symmetrical around $s=7 / 2$ except that Eq. (6) gives greater cross sections for the larger $J$ values.

\section{Conclusion}

We have demonstrated how to systematically find neutron eigenstates in a deformed potential, and use these for $\left({ }^{3} \mathrm{He}, \alpha\right)$ DWBA pickup calculations to find the spin and parity distributions of the residual target nuclei. A simple smoothing convolution was used to take into account the spreading width of the single-neutron hole states into the more complicated compound nuclear states. We proceed by assuming the initial target is an even-even rotor, but can take into account spectator neutrons outside such a rotor by recombining their spin and parity at the end of the calculations. It should be the subject of another project to use our final spin and parity distributions to calculate the gamma-decay or fission probabilities of interest, so that our modeling of this $\left({ }^{3} \mathrm{He}, \alpha\right)$ surrogate reaction can be compared with what is experimentally observed.

\section{Acknowledgements}

This work was performed under the auspices of the U.S. Department of Energy by the University of California, Lawrence Livermore National Laboratory (LLNL) under contract No. W-7405-Eng-48.

\section{References}

[1] J.E. Escher and F.S. Dietrich, Phys. Rev. C 74 (2006) 054601 
[2] B.F. Lyles et al, Bull. Am. Phys. Soc. 51 (2006) 34.

[3] J.M. Bang, F. G. Gareev, W. T. Pinkston and J. S. Vaagen, Physics Reports, 125 (1985) 253

[4] B. Hird, Comp. Phys. Commun. 6 (1973) 30

[5] B. Hird and K.H. Huang, Comp. Phys. Commun. 9 (1975) 293

[6] S. Cwiok, J. Dudek, W. Nazarewicz, J. Skalski and T. Werner, Comp. Phys. Commun. 46 (1987) 379

[7] G.R. Satchler, Ann. Phys. 3 (1958) 275

[8] I.J. Thompson, Pluto: Bound and Scattering States in Deformed Potentials, Draft LLNL Report, 2006.

[9] D. Baye, M. Hesse, J.-M. Sparenberg and M. Vincke, J.Phys. B: At. Mol. Opt. Phys. 31 (1998 ) 3439

[10] M. Hesse, J.-M. Sparenberg, F. Van Raemdonck, D. Baye, Nucl. Phys. A 640 (1998) 37

[11] J. Mortimer, Lagrange mesh technique for solving coupled equations, First Year Report, University of Surrey, July 1999

[12] T. von Egidy, Th. W. Elze and J.R. Huizenga, Nucl. Phys. A145 (1970) 306

[13] W.T. Milner, C.E. Bemis and F.K. McGowan, Phys. Rev. C 16 (1977) 1686.

[14] G.E. Brown and M. Rho, Nucl. Phys. A372 (1981) 397

[15] I.J. Thompson, Fresco. See URL:www.fresco.org.uk. 